\title{
Perfiles de competencias TIC en la práctica educativa ante el reto de la enseñanza remota
}

\author{
ICT competency profiles in the teacher's educational practice to \\ respond to the challenge of remote teaching
}

\section{Perfis de competências em TIC na prática educacional que enfrentam o desafio do ensino à distância}

\author{
Sonia Valbuena Duarte* iD Dariana Rodriguez Gonzalez * iDAndrea Viviana Tavera* * * id \\ * Licenciada en Ciencias de la Educación especialidad en Matemática y Física. Especialista \\ en Física. Maestría en Educación. Maestría en Matemática. Docente Universidad del Atlántico. \\ https://orcid.org/0000-0003-3667-1087. **Licenciada en Matemáticas, Universidad del \\ Atlántico, Puerto Colombia, Colombia. darianadrodriguez@mail.uniatlantico.edu.co ORCID: \\ https://orcid.org/0000-0002-4047-8805. ***Licenciada en Matemáticas, Universidad del \\ Atlántico, Puerto Colombia, Colombia. avtavera@mail.uniatlantico.edu.co ORCID: https://orcid. \\ org/0000-0002-7305-0493.
}

\section{OPEN ACCESS $\boldsymbol{\Omega}$ \\ DOI: http://dx.doi.org/10.18634/sophiaj. 17v.1i.1052}

Información del artículo

Recibido: octubre 08 de 2020

Revisado: febrero 12 de 2021

Aceptado: abril 162021

Publicado: mayo 4 de 2021

Palabras clave: TIC, competencias del docente, práctica profesional, plan de clase, enseñanza remota.

Keywords: ICT, teacher competences, professional practice, lesson plans, remote teaching.

Palavras-chave: TIC, competências do docente, prática profissional, plano de aula, ensino à distância.

Cómo citar: /how cite:

Valbuena, S. (2021) Perfiles de competencias TIC en la práctica educativa ante el reto de la enseñanza remota. Sophia 17 (1) e1052

Sophia-Educación, volumen 17 número 1. enero/junio 2021. Versión español
RESUMEN

Los conocimientos y competencias en el uso de las tecnologías en el sentido didáctico y pedagógico en relación con los impactos en los procesos de enseñanza y aprendizaje han sido tendencia de estudio. Esta investigación centró su atención en describir y examinar perfiles y niveles de apropiación de las Tecnologías de la Información y la Comunicación (TIC) en la práctica profesional del profesor de matemática en función de los usos que desde lo didáctico y pedagógico están presentes en el microcurrículo de este saber disciplinar, la relevancia temática se tiene por las características de la población estudiantil actual y que de manera adicional se devela en estos momentos cruciales de la historia de la humanidad donde el uso de las TIC dejó de ser optativo puesto que debe atender una enseñanza remota. Para tal fin, se recolectó información de profesores de matemáticas de 17 colegios de educación básica y media de la región caribe colombiana a través de entrevistas, grupo focal, observación no participante, revisión documental y análisis didáctico. Del análisis de los resultados se infiere el bajo uso de las tecnologías desde lo pedagógico y didáctico por parte de los profesores integradas en el microcurrículo y a su práctica profesional, evidenciando en su mayoría un uso puramente instrumental de la tecnología en el microcurrículo. Del estudio se logra concluir el poco desarrollo de las competencias TIC en el profesor de matemáticas y aspectos emergentes que posibilitarían optimizar el aprendizaje escolar, complementarios a otros previamente descritos en la literatura. 
Copyright 2021 Universidad La Gran Colombia

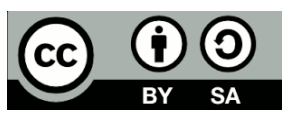

Conflicto de interes:

Los autores declaran no tener ningún cnflicto de interés.

Correspondencia de autor:

soniabalbuena@mail.uniatlantico.edu.co

\section{ABSTRACT}

Knowledge and skills in the use of technologies in the didactic and pedagogical sense in relation to the impacts on the teaching and learning processes have been a trend of study. This research focused its attention on describing and examining profiles and levels of appropriation of Information and Communication Technologies (ICT) in the professional practice of the mathematics teacher based on the uses that from the didactic and pedagogical are present in the microcurriculum of this discipline, the relevance of this study is due to the characteristics of the current student population and additionally because at these crucial moments in human history where the use of ICT is no longer optional because it must attend a remote teaching. This research worked with mathematics teachers from 17 elementary and middle schools in the Colombian Caribbean region collecting information through interviews, focus group, non-participant observation, documentary review, and didactic analysis. From the analysis of the results, it is evident the low use that teachers give to technologies from the pedagogical and didactic point of view in an integrated way in the microcurriculum and their professional practice, and mostly a purely instrumental use of technology in the microcurriculum is identified. From the study it is possible to conclude the little development of ICT skills in the mathematics teacher and emerging aspects that would make it possible to optimize school learning, complementary to previous ones described in the literature.

\section{RESUMO}

Conhecimento e habilidade no uso de tecnologias no sentido didático e pedagógico em relação aos impactos nos processos de ensino e aprendizagem têm sido uma tendência de estudo. Esta pesquisa centrou sua atenção em descrever e examinar perfis e níveis de apropriação das Tecnologias de Informação e Comunicação (TIC) na prática profissional do professor de matemática a partir dos usos que do didático e pedagógico estão presentes no microcurriculum. desta disciplina, a relevância deste estudo deve-se às características da população estudantil atual e, adicionalmente, porque nestes momentos cruciais da história da humanidade onde o uso das TIC já não é opcional porque deve assistir a um ensino à distância. Esta pesquisa trabalhou com professores de matemática de 17 escolas de ensino fundamental e médio da região do Caribe colombiano, coletando informações por meio de entrevistas, grupos focais, observação não participante, revisão documental e análise didática. A partir da análise dos resultados, evidencia-se a baixa utilização que os professores dãoàs tecnologias do ponto de vista pedagógico e didático de forma integrada no microcurriculare na sua prática profissional, sendo sobretudo identificada uma utilização puramente instrumental da tecnologia no microcurricular. A partir do estudo é possível concluir o pouco desenvolvimento de competências em TIC no professor de matemática e aspectos emergentes que possibilitariam otimizar a aprendizagem escolar, complementares às anteriores descritas na literatura. 


\section{Introducción}

La transformación de la sociedad mediada por las Tecnologías de la Información y la Comunicación (TIC) demanda la necesidad de usarlas de forma reflexiva para favorecer la enseñanza y aprendizaje y lograr una educación de calidad, su impacto ha generado que se conciban y se enfoquen como un recurso importante para el desarrollo pedagógico dentro del aula, como factor importante en la enseñanza y aprendizaje (Valencia et al., 2016; Laitón et al., 2017; Valenzuela \& Varela, 2020). El surgimiento de estos recursos requiere del profesor desarrollar competencias, poseer destrezas y conocimientos para usar de forma adecuada las tecnologías (Grisales, 2018; Valenzuela \& Varela, 2020).

Uno de los elementos que gana cada vez mayor importancia en la formación del profesor para la planeación de su propio quehacer en el aula es el modelar secuencias de actividades para lograr objetivos planteados y favorecer el aprendizaje de los estudiantes incluyendo la realidad de estos con una intencionalidad educativa (López \& Villanueva, 2019) y este estado le implica al profesor el reto de hacer uso de las TIC en sus planeaciones para estudiantes que son nativos en la tecnología; sin embargo hay evidencia de que el profesor no se siente confiado con el uso de las TIC en el aula debido a su falta de competencias en estas (Ángel et al., 2016; Ángel \& Patiño, 2018; Valenzuela \& Varela, 2020), y que aún falta mayor reflexión, socialización, y participación en el ámbito académico para que se genere un impacto de las TIC en el microcurriculo (Cantú \& Morado, 2016; Carvajal et al., 2019).

En el campo de la educación, las orientaciones han sido tradicionalmente ajustadas a perspectivas que implican que el profesor en práctica como en formación aprenda a gestionar, conectar y transformar su conocimiento en acciones razonables dentro del aula, de igual manera, su formación en TIC orientada con información que posibilite y facilite el uso de estos recursos para el mejoramiento del aprendizaje de los estudiantes. En general, la apuesta científica de las ciencias de la educación ha implicado la crítica en que Ios profesores no usan las TIC de manera didáctica, evidenciando así una carencia efectiva en el manejo de recursos tecnológicos (Caviedes, Gamboa \& Badillo, 2019; Fernández, Fernández \& Rodríguez, 2018).

Particularmente, en este tema, la educación colombiana a través del Ministerio de Tecnologías de la Información y la Comunicación ha creado distintos programas para que el profesor de básica y media haga uso de las TIC buscando ampliar acceso, utilización y aprovechamiento de las TIC para una educación de calidad, como ejemplo, se encuentra Computadores para Educar (CPE) (2001), Planes TIC (2009), Vive Digital (2010), y ETIC@ (2017). La efectividad de estos programas parece no haber impactado como esperado, ejemplo de ello se reporta en el informe del estudio final de medición y evaluación de CPE (2018) que presentó una apropiación en cuanto a competencias TIC por parte de directivos y docentes y bajo uso de contenidos digitales por parte de profesores. Otro caso en este mismo sentido, se evidencia en la evaluación de impacto del plan Vive Digital realizada por el Departamento Nacional de Planeación (DNP, 2016) que identificó que, no se presentaron efectos positivos en cuanto a las actividades de acceso a educación formal y no formal con uso de TIC.

La relevancia de la utilización desde lo didáctico y pedagógico de los recursos tecnológicos ha sido develada en eventualidades como la que se vive en el mundo en la actualidad a nivel educativo, en la que el uso de la tecnología pasa de ser optativo a ser obligatorio, debido al surgimiento del coronavirus (COVID-19), declarado por la Organización Mundial de la Salud (OMS) como pandemia, el cual es el promotor de que muchos países, entre esos Colombia por medio del Ministerio de Salud y Protección Social (MinSalud), adoptaran como medida preventiva el aislamiento social, por lo que el MEN(2020), aplaza las clases presenciales para abarcarlas de forma virtual o remota.

Este estudio en específico apunta a ofrecer aportes significativos en el análisis del estado del empleo de las TIC de forma pedagógica y didáctica en el microcurrículo y por consiguiente en la práctica profesional del profesor de matemáticas, particularmente en relación con el objetivo de analizar los perfiles y niveles de apropiación (Explorador, Innovador e Integrador) de competencias TIC (Tecnológica, Pedagógica, De gestión, Comunicativa e Investigativa) que poseen los profesores de Matemáticas desde la planeación microcurricular y su práctica profesional, todo ello a partir de la revisión de producción escrita y de trabajo de campo realizado con profesores de educación básica y media en 17 colegios de la ciudad de Barranquilla y algunos municipios de sus alrededores. 


\title{
Marco conceptual
}

\section{La tecnología en el microcurrículo del docente de matemáticas}

La tecnología vista como "actividad humana, que busca resolver problemas y satisfacer necesidades mediante el uso racional y creativo de recursos y conocimientos" (MEN, 2008: 5), la ubica como parte esencial en la planeación curricular (Álvarez \& Blanquicett, 2015), entendido aquí el currículo sobre la base del MEN, del Decreto $230^{1}$ de 2002 en su artículo 2 del capítulo I, como "el conjunto de criterios, planes de estudio, metodologías y procesos que contribuyan a la formación integral y a la construcción de identidad cultural nacional, regional y local, incorporando distintos recursos" (MEN, 2002: 1). En este orden de ideas, las planeaciones de enseñanza organizan el currículo en tres niveles: macrocurrículo (planes de estudio), mesocurrículo (plan de área) y microcurriculo (plan de aula) (Mayora \& Gutiérrez, 2019; MEN, 2017).

En el primer nivel, el plan de estudios definido en la Ley 115 de 1994 o Ley General de Educación en su artículo 79 como el "esquema estructurado de las áreas obligatorias y fundamentales y de áreas optativas con sus respectivas asignaturas, que forman parte del currículo de los establecimientos educativos" (1994: 17). En un segundo nivel, la planificación de cada área que conforma el plan de estudios es "la planificación anual" (MEN, 2017: 21), la ejecución de los objetivos de este plan se organiza en planes de aula por medio de secuencias de actividades "previstas para un periodo temporal limitado" (MEN, 2017: 23), en pro de la calidad del proceso de enseñanza y aprendizaje se proponen cinco momentos desde el MEN. Estos son los siguientes:

\begin{abstract}
Momento de exploración: Se motiva a los estudiantes hacia un nuevo aprendizaje, reconociendo sus saberes previos frente a la temática a abordar y/o la actividad a realizar, la importancia y necesidad de dicho aprendizaje. Momento de estructuración: El docente realiza la conceptualización, introducción de vocabulario y de nuevos procesos, haciendo enseñanza explícita y modelación en relación con el objetivo de aprendizaje. Momento de práctica-ejecución: El docente plantea acciones de aprendizaje que permitan el uso de recursos didácticos como los materiales educativos, con el fin de alcanzar las metas de aprendizaje. Momento de transferencia: El docente planea cómo los estudiantes van a socializar y transferir lo comprendido durante la actividad con el fin de constatar si se logró la meta de aprendizaje. Momento de valoración: el docente realiza el cierre del proceso de aprendizaje que desarrolló generando reflexiones y acciones que permitan responder con los estudiantes a las preguntas: ¿qué se logró? ¿Logramos la meta de aprendizaje? ¿Qué dificultades tuvimos? ¿Qué podríamos mejorar? (MEN, 2017: 25-26).
\end{abstract}

Visto de esta manera, la integración de las TIC al proceso educativo es función clave que todo profesor debe desarrollar en su quehacer (reflejado en su planeación microcurricular), desde la dimensión pedagógica se entiende como una labor y destreza para apoyar una enseñanza que conlleve un aprendizaje lleno de sentido (Valencia et al., 2016), en términos del manejo de recursos tecnológicos implica hacerlo de forma innovadora y creativa para una enseñanza efectiva y asertiva.

\section{La tecnología y su puesta en práctica por el profesor}

En cuanto al profesor, el MEN para el año 2014 documentó las competencias TIC que debe desarrollar: la competencia tecnológica (relacionada con selección y uso pertinente de herramientas tecnológicas), la Comunicativa (es la capacidad para expresarse, establecer contacto y relaciones en espacios virtuales y audiovisuales mediante diversos recursos de manera sincrónica y asincrónica), la Pedagógica (utilizar las TIC para el proceso de enseñanza y aprendizaje con el fin de formar integralmente a los estudiantes), la de Gestión (uso de las TIC de manera adecuada y efectiva en la planeación, organización, administración y evaluación en el ámbito académico), y, por último, la Investigativa (utilizar las TIC para la producción de nuevos conocimientos y la transformación del saber).

Estas competencias TIC son jerarquizadas en tres niveles de desarrollo y complejidad (MEN, 2014), siendo el nivel básico la Exploración, en el que el profesor empieza a introducirse, familiarizarse y reflexionar sobre estos recursos en el proceso de enseñanza y aprendizaje. El siguiente nivel es de Integración; en el que el profesor desarrolla la capacidad de usar la tecnología de forma autónoma y creativa, con ideas que tienen valor a través de la profundización en el proceso educativo, y, por último, el de Innovación, cuando pone en práctica nuevas ideas, construye de manera colectiva nuevos conocimientos y estrategias novedosas con uso de las TIC, de tal manera que permita reconfigurar su práctica.

1. Con el Decreto 230 de 2002, se dictan normas en materia de currículo, evaluación y promoción de los educandos y evaluación institucional, este es derogado por Artículo 19 Decreto 1290 de 2009, en lo referido a reglamentar la evaluación del aprendizaje y promoción de estudiantes de educación básica y media y luego es compilado en el Decreto Único Reglamentario de Educación 1075 de 2015, el cual luego es sustituido en la parte del Capítulo 2 y la supresión del Capítulo 7 del Título 3 de la Parte 5 del Libro 2, a través del Decreto 1330 de 2019. 


\section{Materiales y métodos}

Con enfoque cualitativo y diseño descriptivo (Arias, 2012) la información es obtenida de entrevistas estructuradas a actores involucrados, de revisión documental a microcurrículos de matemáticas, y del análisis didáctico (Rico, 2013) aplicado a libros de textos de Matemáticas, uno de una editorial comercial y otro expedido por el MEN (edición 2017). Se realizó observación no participante y se aplicó también la técnica de grupo focal haciendo registro en lista de control y en diario de campo.

La población de estudio son profesores de básica y media y de ellos se toma una muestra de estudio no probabilística intencional (Arias, 2012) constituida por profesores de Matemáticas vinculados a sendas instituciones de distintos estratos socioeconómicos (1,2 y 3), 13 de instituciones del sector público y 4 de carácter privado, ubicadas en diversas localidades de los municipios de Barranquilla (suroriente, suroccidente, área metropolitana y norte centro-histórico), Soledad (suroriente y suroccidente) y Galapa (suroccidente). En su mayoría los profesores son licenciados en matemáticas a excepción de uno que es ingeniero químico, exceptuando a tres los demás reportan formación continuada, seis cuentan con formación en TIC mediante cursos y diplomados y cuatro mencionan no tener formación en el tema de TIC.

\section{Metodología para el análisis de la información}

La metodología se ejecuta en cuatro fases: 1) Exploración documental, 2) Trabajo de campo, 3) Organización y clasificación de la Información, haciendo uso del diseño de tablas en Word, se organiza y clasifica la información de acuerdo con los niveles de competencias TIC, dimensiones, categorías e indicadores y la fase 4) Análisis de la información, realizada a través de una triangulación entre datos que involucran las consideraciones recogidas y lo aportado por los referentes teóricos. También se hace uso del análisis didáctico adaptado de Rico (2013), teniendo en cuenta 4 de los 5 ciclos. Así el análisis se realiza en tres frentes:

En la primera parte se analizan los microcurrículos de los profesores de matemáticas que hacen parte de la muestra en aspectos pedagógicos y didácticos del uso de la tecnología, los momentos que componen su plan de aula y los niveles de competencias TIC identificados en los que se encuentra el profesor.

El segundo frente de análisis se basa en indicadores que den cuenta de conocimiento de las TIC, uso en las planeaciones de aula y creación de contenidos digitales por parte del profesor, asociando estos con los niveles de desarrollo de las competencias TIC y se identifica a partir de sus desarrollos en que generación es posible ubicarle.

Finalmente, el tercer frente de análisis usando el Análisis didáctico adaptado de Rico (2013) en 4 ciclos, Análisis de contenido, Análisis cognitivo, Análisis de instrucción y Análisis de evaluación buscando información del apoyo para el profesor en el desarrollo y perfeccionamiento de competencias TIC a través de recursos bibliográficos; representado en los libros de texto que utiliza.

\section{Resultados}

\section{Análisis de los microcurriculo de Matemáticas}

Los microcurrículos de los profesores de matemáticas que hacen parte de la muestra se analizan desde el uso pedagógico y didáctico que dan a la tecnología, los momentos que componen las planeaciones de los profesores, y los niveles de competencias TIC en los cuales se encuentra el profesor, teniendo claro que pueden darse niveles de desarrollo diferentes para cada competencia.

El análisis entorno a las dimensiones pedagógica y didácticas es adaptado de Valencia et al. (2016) y de Ángel y Patiño (2018) usando criterios como recursos didácticos interactivos, uso de instrumentos y concertación de criterios de evaluación en línea, uso de fuentes de información, normas de citación y referenciación, conocimiento de plataformas e-learning y recursos Web y la preparación de material didáctico con TIC.

Siendo así, se evidencia que 7 de los 17 profesores de la muestra se comunican, navegan y usan fuentes de información con uso de TIC e incluyen recursos tecnológicos como YouTube, programas computacionales y páginas web en sus espacios profesionales y personales y le reconocen el valor educativo que pueden ofrecer. Sin embargo, al analizar los microcurrículos diseñados para el año 2019 y parte del 2020, es posible dividir los profesores en dos grupos: el primero, con una cantidad considerable que no incluyen recursos de esta naturaleza en sus planeaciones y un segundo grupo conformado por solo tres profesores que hacen 
algún uso de este tipo de recursos y, dentro de este grupo, en dos de ellos no hay evidencia específica de la intencionalidad pedagógica y didáctica de uso, dejando ver un uso meramente instrumental del recurso.

Lo presentado anteriormente conlleva a identificar al primer grupo de profesores con un perfil explorador en relación con las competencias tecnológica, pedagógica, de gestión y comunicativa, debido a que reconocen diversas herramientas tecnológicas y algunas formas de integrarlas, observándose un uso preponderante como medio de comunicación con sus estudiantes por redes sociales y correos electrónicos y el uso de internet para búsqueda de información. En el segundo grupo de profesores, se les logra ubicar un perfil con nivel integrador con respecto a las competencias tecnológica y pedagógica ya que usan diversos recursos TIC en diferentes contextos educativos y presentan algunas estrategias pedagógicas para potenciar el aprendizaje del estudiante. Ningún profesor es ubicado en estas competencias con perfil en el nivel innovador, dado que no se evidencia que diseñen ambientes de aprendizaje o de gestión institucional con uso de herramientas TIC especializadas, y no adaptan nuevos modelos e ideas de diversas fuentes. Además, algunos profesores de instituciones públicas en la localidad suroriente y norte-centro histórico no integran ningún recurso TIC por lo que no es posible ubicarlos con algún perfil ni nivel de apropiación en tecnología.

Es de anotar, no obstante, que en las planeaciones de aula (de abril y mayo de 2020), en tiempo posterior a la declaración de realización de clases desde un acceso remoto, se encontró que los profesores de las instituciones privadas en las localidad norte-centro histórico, suroriente y suroccidente, incluyen recursos tecnológicos como plataformas educativas, redes sociales, Zoom y YouTube para retroalimentar y desarrollar habilidades de ejercitación en el estudiante , y de estos solo los profesores en la localidad norte-centro histórico y suroriente diseñan actividades con uso de recursos TIC para valorar, orientar y potenciar el aprendizaje (un ejemplo es mostrado en Figura 1).

Figura 1. Actividad diseñada por profesor y visualizada en plataforma digital

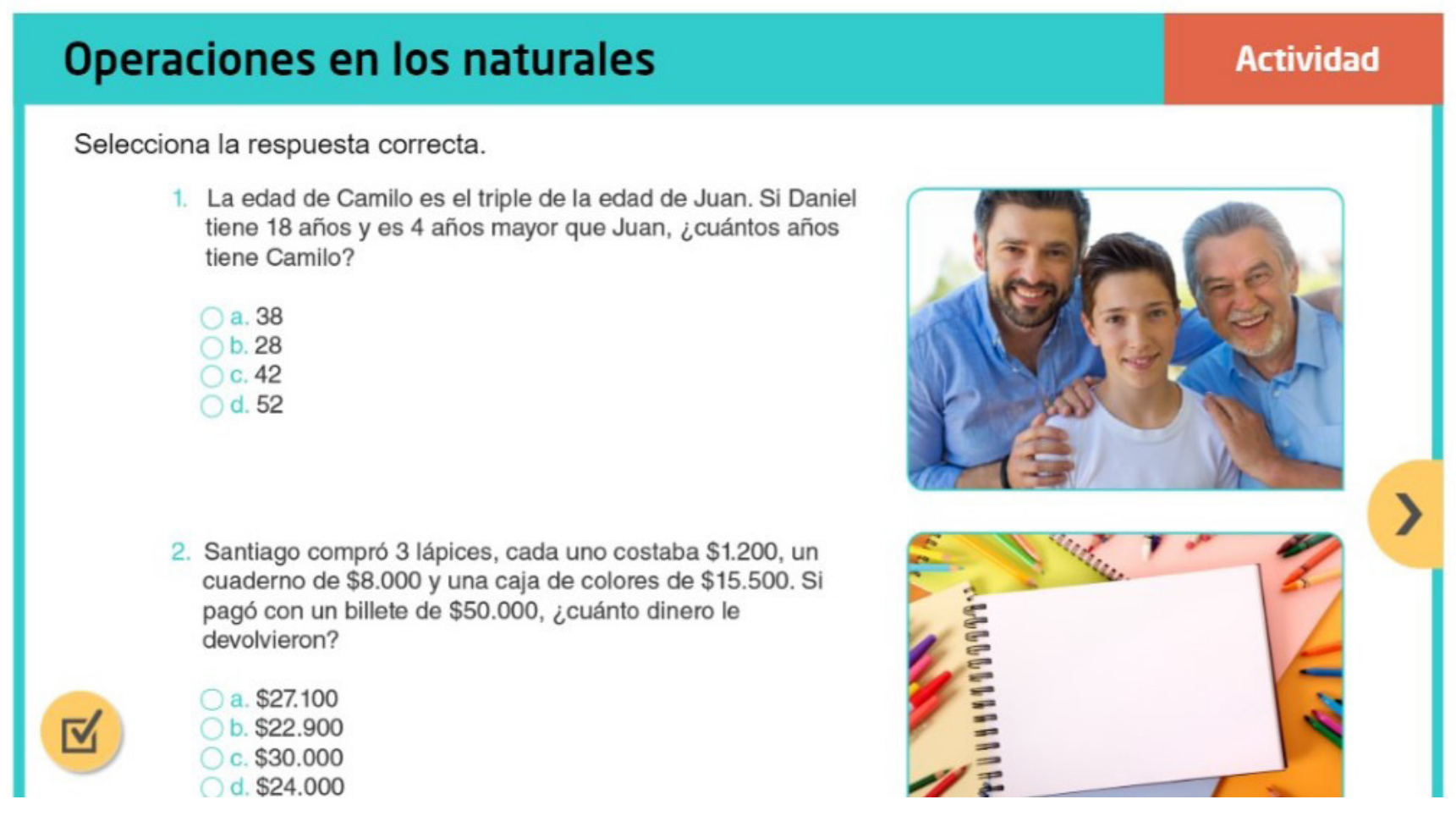

Fuente: propia de observación a profesor de institución en localidad norte-centro histórico

En las planeaciones de aula diseñadas para la enseñanza de manera remota es identificado solo en algunos profesores de instituciones públicas (localidad norte-centro histórico) y en todos los de instituciones privadas que en el momento exploración usan videos de la plataforma YouTube para ampliar información de la temática dada, mapa de ideas y plataformas virtuales.

Y en el momento de estructuración, los profesores de instituciones públicas y privadas en la localidad metropolitana, suroriente y suroccidente, para la introducción de temas nuevamente incluyen videos de la plataforma YouTube para complementar conceptos y definiciones. En el momento de práctica-ejecución, siete profesores no realizan actividades usando como recursos la tecnología, gran parte de ellos incluyen actividades 
del texto guía para realizar de forma manual, a excepción de los profesores de instituciones privadas en norte-centro histórico, y un profesor de institución pública en suroriente, utilizan talleres virtuales con la intencionalidad de valorar lo aprendido por el estudiante. Para el momento de transferencia, solo los profesores de instituciones privadas de norte-centro histórico apoyándose en redes sociales y plataformas retroalimentan, orientan y valoran el aprendizaje de los estudiantes. Por último, en el Momento de valoración solo cuatro profesores realizan preguntas sobre aspectos a mejorar y dificultades que tuvieron los estudiantes, pero ningún profesor utiliza recursos tecnológicos que puedan apoyar a que se dé una reflexión del proceso de aprendizaje.

\section{Análisis de los cuestionarios aplicados a los profesores de Matemáticas}

De los cuestionarios; diario de campo y lista de verificación aplicados en tiempos de enseñanza remota a toda la muestra se han analizado indicadores que den cuenta de conocimiento de las TIC, su uso en las planeaciones de aula y creación de contenidos digitales, asociando estos con los niveles de desarrollo de las competencias TIC.

Con relación al primer indicador del dominio de herramientas tecnológicas educativas en el diseño e impartición de clases, aunque se evidencia que en 10 de 17 profesores el uso de diversos recursos tecnológicos como páginas web, redes sociales, plataformas educativas y programa computacional, no se evidencia la intencionalidad didáctica de uso, y en siete profesores no se encuentran uso de recursos TIC, lo que no muestra un notable reconocimiento del valor epistémico y didáctico de su uso. Los hallazgos en este grupo de profesores objeto de estudio son concordantes con trabajos previos con población similar (Carvajal et al., 2019; Padilla \& Conde, 2019; Palos, Gómez \& Alemán, 2017; Teliz, 2015) pues, aunque los profesores tienen una percepción positiva sobre el uso de las TIC en las prácticas de enseñanza, persiste la no integración al proceso educativo, existiendo un escaso uso didáctico de las mismas con propósitos significativos en el aula de clase.

Con relación al indicador planificación de clases con uso de TIC, relacionados con el nivel integrador se identifican seis profesores en este nivel en la competencia tecnológica y en igual nivel en la de gestión debido a que combinan variedad de recursos tecnológicos para mejorar la planeación de sus prácticas educativas ya que acceden a programas de formación apropiados para necesidades de desarrollo. En lo referente a la competencia pedagógica, investigativa y comunicativa no se evidenció este nivel en los profesores pues no hubo hallazgos de liderazgo, presentaciones o desarrollos de proyectos ni estrategias de aprendizaje en el contexto escolar usando TIC.

Otro aspecto encontrado con respecto a la planeación de clase con uso de las TIC, radica en que una cantidad importante de los profesores al integrar la tecnología no explicitan el uso desde lo didáctico y pedagógico y le dan un uso básico a la tecnología en el microcurrículo, faltando mayor reflexión, comprensión y participación para que las TIC se pudiera llegar a impactar lo microcurricular; lo que difiere a lo proyectado por el MEN (2017) pues lo esperado es que el profesor en sus planes de aula integre intencionalmente materiales (libros de texto, recursos audiovisuales, libros de la biblioteca escolar, entre otros), involucrando diferentes estilos de aprendizaje de los estudiantes, contando con herramientas TIC y recursos didácticos desarrollados a partir del contexto.

Por último, en el criterio usa o crea medios digitales específicos para dar significado a contenidos matemáticos, es relacionado con el nivel innovador y se evidencian profesores ubicados en este nivel en relación con la competencia tecnológica en cuanto al uso de recursos de esta naturaleza para construir aprendizajes en sus estudiantes, y un poco menos de la mitad de la muestra se ubican en este mismo nivel en la competencia pedagógica ya que diseñan ambientes de aprendizaje y los evalúan en ambos casos poniendo en funcionamiento diferentes estrategias mediadas por las TIC, como quices, foros y talleres virtuales, por medio de plataformas educativas (Class notebook, Microsoft Teams, Zoom, entre otras), y una docente plantea que utiliza recursos como el televisor, y radio, para generar discusiones y conexiones con estudiantes,. En cuanto a las competencias, comunicativa, investigativa y de gestión no se logró evidenciar a ningún profesor en este nivel.

En general los hallazgos develan el que los profesores con frecuencia incluyen muy poco las TIC como recurso didáctico en sus prácticas de enseñanza o en algunos casos hay ausencia de esta inclusión lo cual es un aspecto similar al encontrado en estudios previos (Ángel \& Patiño, 2018; Fernández, Fernández y Rodríguez, 2018; Gutiérrez, Aristizábal, \& Rincón, 2020; Teliz, 2015; Valenzuela \& Varela, 2020), adicionalmente como hallazgo a reportar se encuentra con alta frecuencia que el profesor no explicita la intencionalidad pedagógica 
y didáctica de uso de estos recursos, lo que parece haber volcado a que los profesores en estos tiempos de enseñanza remota solamente usen las TIC mayoritariamente como medio de comunicación y no con todo el potencial que estos recursos tienen para el desarrollo de procesos de pensamiento en el estudiante. Emerge así, en este estudio un aspecto importante y es el deseo de los profesores del conocimiento para generar, desarrollar y potenciar en el estudiante aprendizaje colaborativo y demás acciones que le conlleven a aprendizajes significativos con desarrollo de pensamiento crítico y demás habilidades de pensamiento de orden superior haciendo uso de las TIC.

Finalmente, con base en los hallazgos descritos es posible ubicar un gran número de ellos en la llamada educación basada en TIC o tercera generación y unos pocos en la cuarta generación llamada educación basada en web, de las cinco generaciones planteadas por Yong et al. (2017). Siendo educación interactiva la quinta generación, en la cual ningún profesor de la muestra es ubicado. Aspecto similar estudiado por Peláez et al. (2018) en Ecuador, Fernández, Fernández y Rodríguez (2018) en España, y Palos, Gómez y Alemán (2018) en México lo cual lleva a considerar que los profesores de la muestra se encuentran inmersos en la generación cuarta. En 2016, Gazcón, Larregui y Castro (2016) en Argentina reportan profesores con una perspectiva en cuanto al uso de libros usando realidad aumentada y la reconstrucción 3D para introducir conceptos espaciales y geométricos, ubicándolos así en la generación cinco. Aspecto que no se encontró en la muestra objeto de estudio y lo que ubicaría al profesor en un nivel de competencias innovador.

\section{Análisis didáctico}

Con el objetivo de analizar el apoyo en recursos bibliográficos que tiene el profesor en cuanto a su desarrollo y perfeccionamiento de competencias TIC, se hace análisis didáctico en cuatro ciclos adaptados de Rico (2013); análisis de contenido, análisis cognitivo, análisis de instrucción y análisis de evaluación a dos textos de matemáticas utilizados por los docentes en las instituciones objeto de estudio: Caminos del saber 10 de la editorial Santillana edición 2013 (texto 1) y Matemáticas 10 del Gobierno Nacional de Colombia edición 2017 (texto 2).

En el análisis de contenido, en lo referente a lo estructural y representacional, el texto 1 usa formatos multimedia para presentar definiciones y conceptos y solo al final de dos unidades presenta una sección corta donde se integra programas computacionales especializados (Microsoft Mathematics, Wolframalpha, Graph, GeoGebra y Excel) donde solo uno es de uso libre (GeoGebra), la Figura 2 ejemplifica. En cada actividad el libro presenta indicaciones, objetivos de usos y actividades. El texto 2, en tres unidades presenta un contenido que titula MatemaTICS usando GeoGebra para construir gráficas, sin especificar objetivos e intencionalidad didáctica de uso.

Figura 2. Programas computacionales integrados en el texto 1

\section{Trabaja con GeoGebra}

Objetivo: construir la gráaica de la función seno a partir de la circunferencia unitaria, identificando sus valores para distintos ángulos.

Descripción: construir la circunferencia unitaria y un ángulo en posición normal, para construir la gráfica de la función seno, utilizando herramientas de GeoGebra como el deslizador. Luego, identificar los valores de la función seno para distintos ángulos.

Para acceder a GeoGebra, ingresa y descarga el programa en www.geogebra.org/cms/es

1 Haz clic en GeoGebra en el menú Inicio.

2) En la barra de herramientas, haz clic en Circunferencia dados su Centro y uno de sus Puntos.

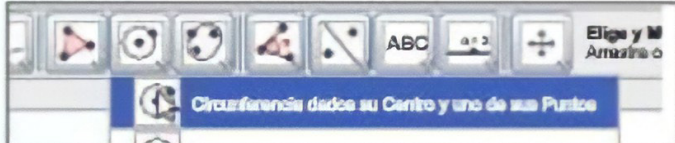

3 En el plano cartesiano haz clic en -1 y en 0
(6) Ubica cuatro puntos en el plano cartesiano, como se muestra en la siguiente imagen.

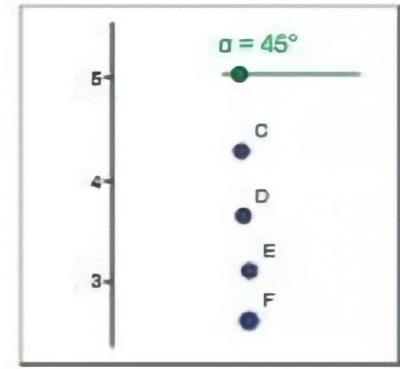




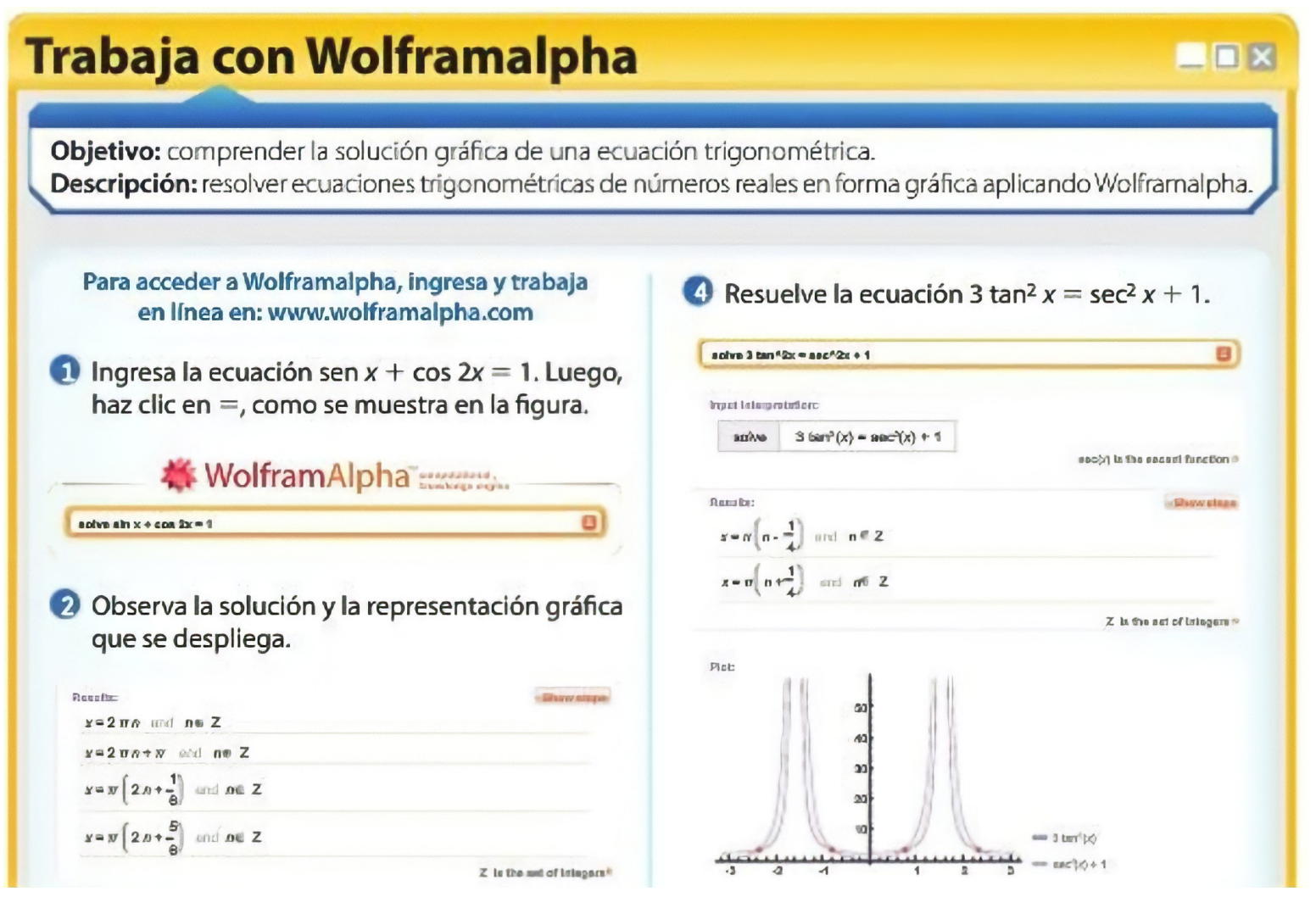

Fuente: Imagen de actividad de texto 1

Del análisis cognitivo, en lo referente a demandas cognitivas y expectativas sobre el aprendizaje los dos libros integran recursos tecnológicos para algunas actividades para afianzar las competencias interpreto, argumento, propongo, ejercito y soluciono problema, no evidencian integración de recursos TIC para tratar posibles dificultades de aprendizaje.

En cuanto al análisis de instrucción, los materiales y recursos; en el texto hace uso de varios programas computacionales para resolver y afianzar actividades, mientras en el texto 2 usa un programa computacional (GeoGebra) y la calculadora científica (Figura 3).

Figura 3. Programa computacional integrado en el texto 2

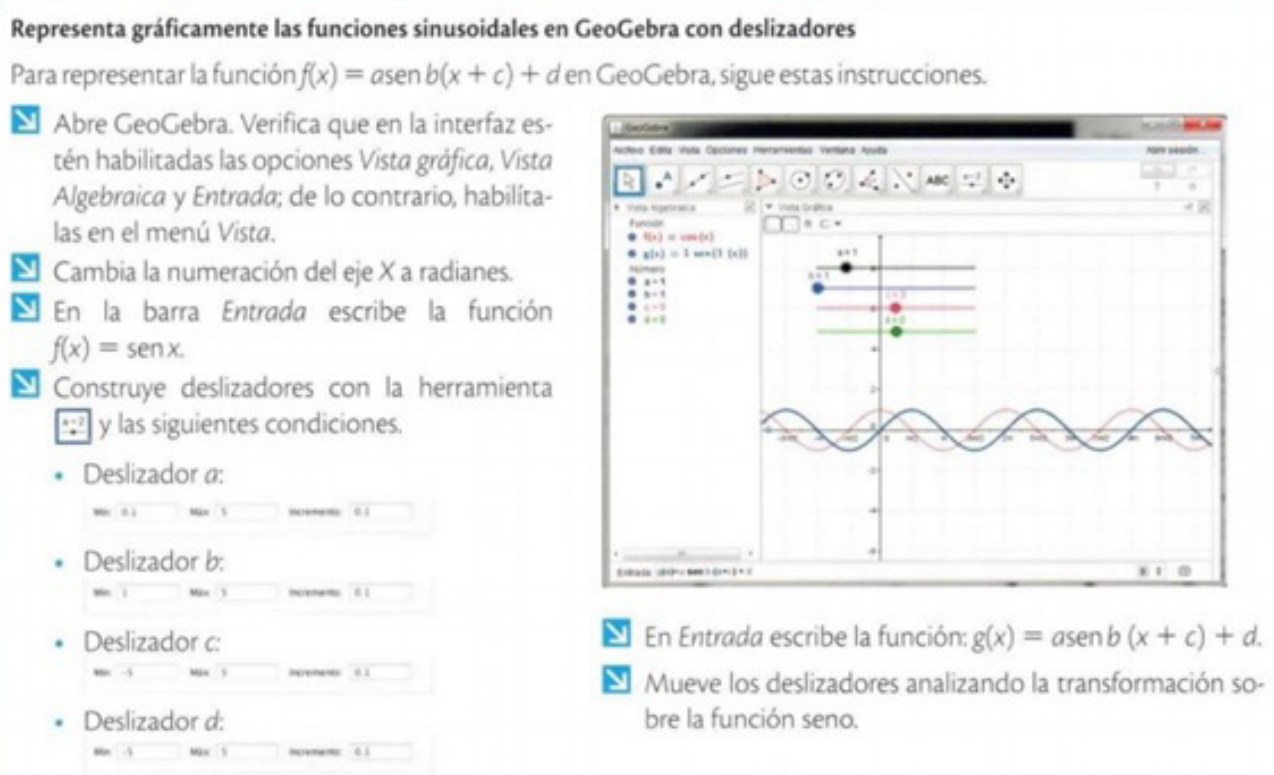

$\$$ En Entrada escribe la función: $g(x)=\operatorname{asen} b(x+c)+d$.

$\square$ Mueve los deslizadores analizando la transformación sobre la función sena 


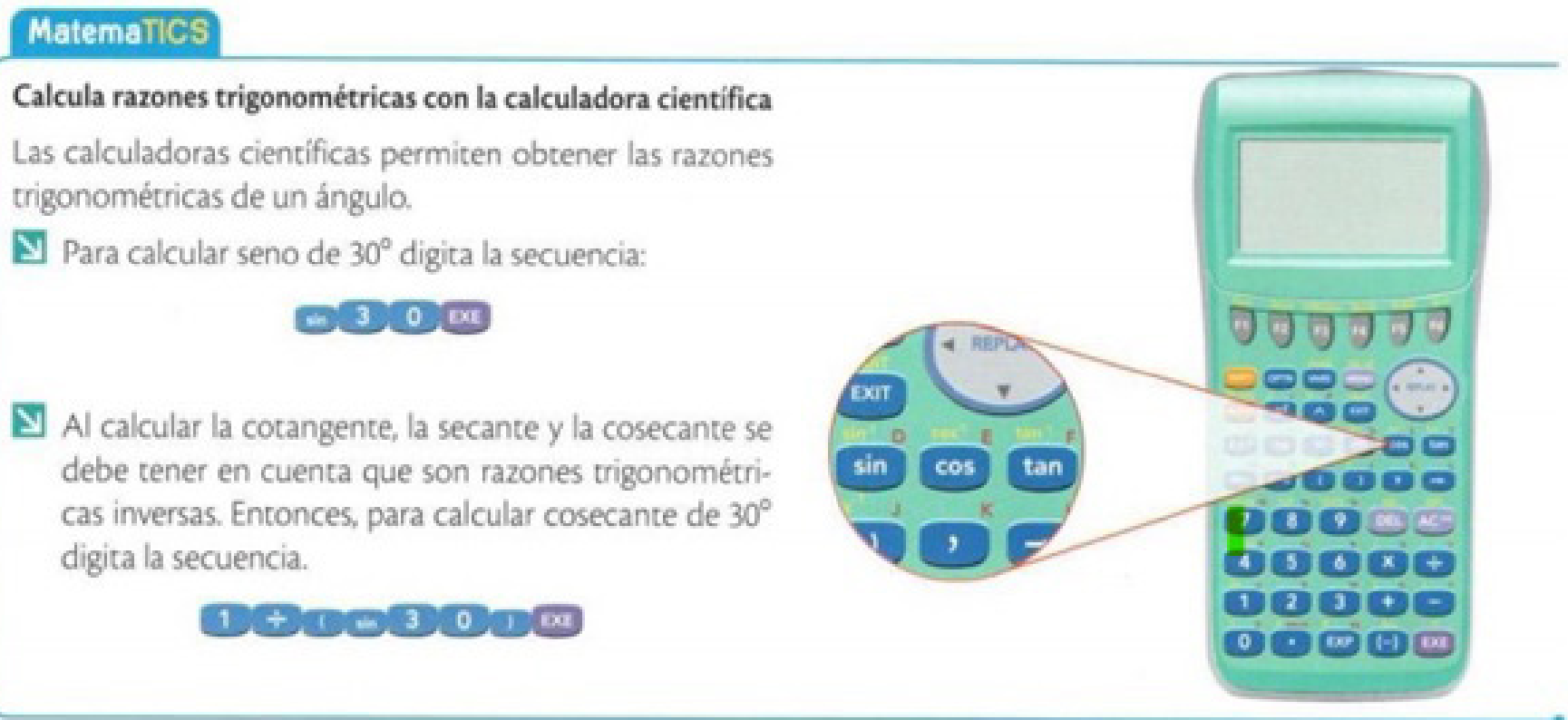

Fuente: Imagen de actividad de texto 2.

Finalmente, en el análisis de evaluación la constante identificada en ambos textos son actividades sin el uso de TIC.

\section{Conclusiones}

Llama la atención la notable divergencia entre el discurso de la mayoría de los profesores con lo observado en su práctica profesional, notándose que reconocen la relevancia de los recursos TIC, su importancia y posibilidades que brindan a la educación y sin embargo, es marcada la carencia de su uso en las planeaciones de aula elaboradas antes de los periodos de inicio de las clases remotas, siendo muy pocos los casos donde se encuentra su uso y lo frecuente es basar el desarrollo de sus actos pedagógicos en los textos y guías físicas y clases magistrales sin uso de recursos TIC. Los resultados obtenidos son consistentes con la evidencia empírica previa, la cual destaca la notable tendencia por el no uso de las TIC en los procesos educativos (Valencia et al., 2016; Laitón et al., 2017; Valenzuela \& Varela, 2020) o bien, entre aquellos que sí los emplean, es común el uso de procedimientos de baja complejidad principalmente de naturaleza instrumental, este hallazgo es reiterativo en los microcurrículos en tiempo de enseñanza remota. Es claro que el uso de estos recursos requiere desarrollar competencias, poseer destrezas y conocimientos para usar de forma adecuada las tecnologías (Grisales, 2018; Valenzuela \& Varela, 2020), por lo cual la mayoría de los microcurrículos estudiados diseñados por los profesores de la muestra poseen un uso desde lo pedagógico y didáctico de las TIC en nivel exploratorio en las competencias estudiadas.

Se concluye así, que falta mayor reflexión por parte de los profesores para que las TIC impacten lo microcurricular y su práctica profesional pedagógica, además de la necesidad de desarrollar competencias TIC en los profesores y estos a su vez en sus estudiantes en cuanto al saber disciplinar estudiado. De trabajarse en el desarrollo de estas competencias TIC e integrarlas a los procesos educativos de manera que apoyen desde lo pedagógico y didáctico todo el proceso, el profesor avanzaría en sus procesos de apropiación de las TIC, desarrollo y perfeccionamiento de estas competencias de forma tal que en cualquier tiempo, incluido eventualidades como uso de enseñanza remota para la educación presencial, las planificaciones de aula estarían diseñadas con recursos de tal manera que con uso adecuado de la tecnología se potencie el aprendizaje del estudiante como planteado por algunas resultados previos (Ángel \& Patiño, 2018; Sullivan et al., 2020; Trouche et al., 2020) y no tratar de forzar y replicar los procesos de una "presencialidad" en una enseñanza remota.

Por su parte, los hallazgos de este estudio presentan una diversidad de elementos que resaltan la importancia que tienen las TIC y la implementación de diversas estrategias con uso de estos recursos para la enseñanza y aprendizaje de las matemáticas, permitiéndole fomentar un aprendizaje colaborativo, e innovar en el 
aula, generando cambios en su práctica, lo cual ha mostrado coincidencias con trabajos previos (Carvajal et all., 2019). Del mismo modo, los aspectos emergentes en este estudio resultan complementarios a los previamente descritos en la literatura, resaltando la posibilidad de optimizar el aprendizaje escolar, desarrollar el pensamiento crítico en estudiantes, desarrollos en la investigación en educación matemática en lo referido a la tecnología como mediador real y efectivo del proceso educativo, no como una eventualidad sino como una práctica usual y cotidiana que desarrolle pensamiento y competencias en el profesor y este a su vez en sus estudiantes y en un panorama de análisis amplio otro aspecto emergente es el uso de las TIC desde lo disciplinar.

Los dos libros de texto analizados hacen poco uso de recursos TIC y para cuando lo usan recae mayoritariamente en programas computacionales especializados, lo que puede convertirse en apoyo al profesor para el desarrollo de competencias de esta naturaleza, sin embargo estos programas son utilizados con marcado énfasis en agilizar cálculos sobre el desarrollo de procesos de aprendizaje y dado que solo uno de los cuatro programas computacionales utilizados en el texto de editorial comercial es de uso libre (gratuito) debilita sustancialmente la fortaleza de estos recursos para desarrollar procesos educativos. Esta perspectiva hace emerger otro aspecto relacionado con la imperiosa necesidad de elevar las competencias del docente en TIC más allá de un uso meramente instrumental de forma que lo ubique en desarrollos a nivel de innovador.

En síntesis, es deseable que el profesor tenga uso adecuado del conocimiento específico de las TIC desde su uso didáctico y pedagógico lo que fomenta un eficaz desempeño profesional (Llinares, 2012; Pochulu, Font, y Rodriguez, 2016) y además que todo recurso material le contribuya a fomentar y fortalecerse en las competencias TIC.

\section{Referencias bibliográficas}

Álvarez, G., \& Blanquicett, J. (2015). Percepción de los docentes rurales sobre las TIC en sus prácticas pedagógicas. Ciencia, Docencia y Tecnología. 26(51), 371-394. En: https://www.redalyc.org/ pdf/145/14542676016.pdf

Ángel, I., \& Patiño, M. (2018). Línea base de indicadores de apropiación de TIC en instituciones educativas. Educación y Educadores, 21(3), 435-457. doi: http://dx.doi.org/10.5294/edu.2018.21.3.4

Ángel , J., Prat, M., Perez, A., \& Steegman, C. (2016). MATH-ELEARNING@CAT: Factores claves del uso de las TIC en Educación Matemática secundaria. Revista Latinoamericana de Investigación en Matemática Educativa, 19(3), 287-310. doi: http://dx.doi.org/10.12802/relime.13.1932

Arias, F. (2012). El proyecto de investigación introducción a la metodología científica. 6. ${ }^{a}$ edición. Editorial Episteme. Caracas, Venezuela.

Cantú, M., \& Morado, C. (2016). Planes de clase de historia: Un momento en la formación Docente. Opción, 32, No. Especial (11), 228 - 246. En: https://produccioncientificaluz.org/index.php/opcion/article/view/21944

Carvajal, S., Giménez, J., Font, V., \& Breda, A. (2019). La competencia digital en futuros profesores de matemáticas. En Badillo, E., Climent, Fernández, C., \& González, M.(Eds) Investigación sobre el profesor de matemáticas: formación, practica de aula, conocimiento y competencia profesional. 285-306. Salamanca: Ediciones Universidad de Salamanca.

Caviedes, S., Gamboa, G., \& Badillo, E. (2019). Conexiones Matemáticas que establecen futuros maestros de primaria en tareas de media y comparación de áreas. Praxis, 15(1), 69-87. doi: http://dx.doi. org/10.21676/23897856.2984

CPE. (2018). Informe final del estudio de medición y evaluación de impacto de CPE 2014-2018. Obtenido de Computadores Para Educar. En: http://computadoresparaeducar.gov.co/sites/default/files/inline-files/ Informe\%20final\%20del\%20estudio\%20de\%20medicion\%20y\%20evaluacion\%20de\%20impacto\%20 de\%20CPE\%202014\%202018.pdf

DNP (2016). Evaluación de Impacto del Plan Vive Digital. Dirección de Seguimiento y Evaluación de Políticas Públicas. En: https://colaboracion.dnp.gov.co/CDT/Sinergia/Documentos/Plan_Vive_Digital.pdf 
Fernández, F., Fernández, M. J., \& Rodríguez, J. (2018). El proceso de integración y uso pedagógico de las TIC en los centros educativos madrileños. Educación XX1, 21(2), 395-416. doi: http://dx.doi.org/10.5944/ educXX1.17907

Gazcón, N., Larregui, J., \& Castro, s. (2016). La realidad aumentada como complemento motivacional. Libros aumentados y reconstrucción 3D. Revista Iberoamericana de tecnología en Educación y Educación en Tecnología.17, 7-15. En: https://dialnet.unirioja.es/servlet/articulo?codigo=5623644

Grisales, A. M. (2018). Uso de recursos TIC en la enseñanza de las matemáticas: retos y perspectivas. Entramado, 14(2), 198-214. doi: http://dx.doi.org/10.18041/1900-3803/entramado.2.4751

Gutiérrez, H., Aristizabal, J.H., Rincón, J.A. (2020) Procesos de visualización en la resolución de problemas de matemáticas en básica primaria apoyados en ambientes de aprendizaje mediados por las TIC. Sophia, 16(1), 120-132. doi: http://dx.doi.org/10.18634/sophiaj.16v.1i.975

Laitón, E., Gómez, S., Sarmiento, R., \& Corredor, C. (2017). Competencia de prácticas inclusivas: Las TIC y la educación inclusiva en el desarrollo profesional docente. Sophia, 82-95. doi: http://dx.doi.org/ 10.18634/ sophiaj.13v.2i.502

Llinares, S. (2012). Construcción de conocimiento y desarrollo de una mirada profesional para la práctica de enseñar matemáticas en entornos en línea. Avances de investigación en Educación Matemática, 2, 53-70. En: http://rua.ua.es/dspace/handle/10045/25111\#vpreview

López, L. \& Villanueva, O. (2019). La atención selectiva del docente en los procesos de planeación curricular, aprendizaje y evaluación. Diálogos sobre educación temas actuales en investigación educativa, 19(10), 1-23. doi: http://doi.org/10.32870/dse.v0i19.489

Mayora, C., \& Gutíerrez, D. (2019). Análisis del currículo del área de inglés en escuelas oficiales de Cali. Lenguaje, 47(25), 685-707. Em: https://revistalenguaje.univalle.edu.co/index.php/lenguaje/\%20 article\%20/view\%20/7519

MEN. (2002). Decreto 230. Por el cual se dictan normas en materia de currículo, evaluación y promoción de los educandos y evaluación institucional. Obtenido de Ministerio de Educación Nacional: http://structio. sourceforge.net/leg/dec230110202.pdf

MEN. (2008). Guía numero 30 ser competente en tecnología: juna necesidad para el desarrollo! Obtenido de Ministerio de Educación Nacional: https://www.mineducacion.gov.co/1759/articles-160915_archivo_pdf. pdf

MEN. (2014). Competencias TIC para el desarrollo profesional docente. Obtenido de Ministerio de Educacion Nacional: https://www.mineducacion.gov.co/1759/articles-339097_archivo_pdf_competencias_tic.pdf

MEN. (2017). Guía de fortalecimiento curricular. Obtenido de Colombia Aprende: https://aprende. colombiaaprende.edu.co/sites/default/files/naspublic/guia_fortalecimiento_curricular.pdf

MEN. (2020). Circular número 020. Obtenido de Ministerio de Educación Nacional: https://www.mineducacion. gov.co/1759/articles-394018_recurso_1.pdf

Padilla, E. I., \& Conde-Carmona, R. J. (2020). Uso y formación en TIC en profesores de matemáticas: un análisis cualitativo. Revista Virtual Universidad Católica del Norte, 60, 116-136. doi: https://www.doi. org/10.35575/rvucn.n60a7

Palos, A., Gómez, M., \& Alemán, L. (2017). Innovación y TIC en docentes de matemáticas en nivel medio superior. Eduweb, 11(1), 89-99. En: https://dialnet.unirioja.es/servlet/articulo?codigo=6638744

Peláez, R., Morales, J., Lara, C., \& Tumbaco, M. (2018). Las TIC y el uso de EVEA en instituciones de educación básica en Guayaquil-Ecuador. Revista Lasallista de investigación, 131-140. doi: https://www.doi. org/10.22507/rli.v15n2a10

Pochulu, M., Font, V., \& Rodríguez, M. (2016). Desarrollo de la competencia en análisis didáctico de formadores de futuros profesores de matemática a través del diseño de tareas. Revista Latinoamericana de 
investigación en Matemática Educativa, 71-98. doi: https://www.doi.org/10.12802/relime.13.1913

Rico, L. (2013). El Método del Análisis Didáctico. Revista Iberoamericana de Educación Matemática, 33, 11-27.

Sullivan, P., Bobis, J., Downton, A., Feng, M., Hughes, S., Livio, S., Russo, J. (2020). Threats and opportunities in remote learning of mathematics: implication for the return to the classroom. Mathematics Education Research Journa doi: https://doi.org/10.1007/s13394-020-00339-6

Teliz, F. (2015). Uso didáctico de las TIC en las buenas prácticas de enseñanza de las matemáticas. Estudio de las opiniones y concepciones de docentes de educación secundaria en el departamento de Artigas. Cuadernos de Investigación Educativa, 13-31. doi: http://dx.doi.org/10.18861/cied.2015.6.2.34

Trouche, L., Rocha, K., Gueudet, G., \& Pepin, B. (2020). Transition to digital resources as a critical process in teachers' trajectories: the case of Anna's documentation work. ZDM: The International Journal On Mathematics Education. Springer. doi: https://doi.org/10.1007/s11858-020-01164-8

Valencia, T., Serna, A., Ochoa, S., Caicedo, A., Montes, J., \& Chávez, J. (2016). Competencias y estándares TIC desde la dimensión pedagógica: una perspectiva desde los niveles de apropiación de las TIC en la práctica educativa docente. Cali: Javeriano-Pontificia Universidad Javeriana-Cali. En: http://www.unesco.org/new/ fileadmin/MULTIMEDIA/FIELD/Santiago/pdf/Competencias-estandares-TIC.pdf

Valenzuela, J., \& Varela, S. (2020). Uso de las tecnologías de la información y la comunicación como competencia transversal en la formación inicial de docentes. Revista Electrónica Educare, 24, 1-20. doi: http://doi.org/10.15359/ree.24-1.10.

Yong, E., Nagles, N., Mejia, C., \& Chaparro, C. (2017). Evolución de la educación superior a distancia: desafíos y oportunidades para su gestión. Revista virtual Universidad Católica del Norte, 80-105. doi:0124-5821 